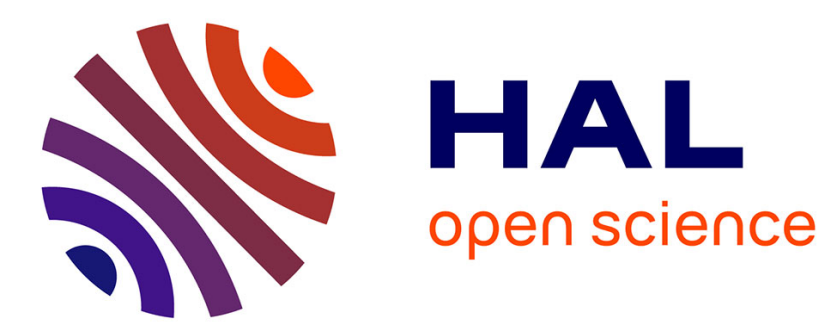

\title{
Une théorie des réseaux des troubles mentaux
}

Denny Borsboom

\section{- To cite this version:}

Denny Borsboom. Une théorie des réseaux des troubles mentaux. 2021, pp.86 - 94 . 10.1016/j.amp.2020.11.014 . hal-03213320

\section{HAL Id: hal-03213320 \\ https://hal.science/hal-03213320}

Submitted on 11 May 2021

HAL is a multi-disciplinary open access archive for the deposit and dissemination of scientific research documents, whether they are published or not. The documents may come from teaching and research institutions in France or abroad, or from public or private research centers.
L'archive ouverte pluridisciplinaire HAL, est destinée au dépôt et à la diffusion de documents scientifiques de niveau recherche, publiés ou non, émanant des établissements d'enseignement et de recherche français ou étrangers, des laboratoires publics ou privés. 


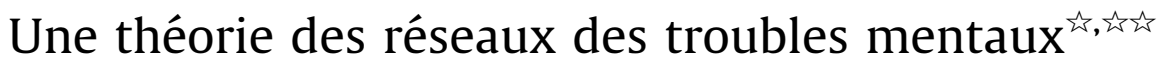

\section{A network theory of mental disorders}

\author{
Denny Borsboom \\ Département de psychologie, université d'Amsterdam, 1018 XA Amsterdam, Pays-Bas
}

\section{N F O A R T I C L E}

\section{Historique de l'article :}

Disponible sur Internet le 25 décembre 2020

\section{Mots clés :}

Approche réseau

Psychopathologie

Réseaux de symptômes

Résilience

Diagnostic

Santé mentale

Traitement

Troubles mentaux

Vulnérabilité

\begin{abstract}
R É S U M É
Ces dernières années, l'approche réseau de la psychopathologie a été proposée comme moyen alternatif de conceptualiser les troubles mentaux. Dans cette approche, les troubles mentaux résultent d'interactions directes entre les symptômes. Bien que l'approche réseau ait conduit à de nombreuses nouvelles méthodologies et à des applications notables, elle n'a pas encore été entièrement formulée en tant que théorie scientifique des troubles mentaux. L'article présent vise à développer une telle théorie, en postulant un ensemble limité de principes théoriques concernant la structure et la dynamique des réseaux de symptômes. La notion de lien causal, constituée par les symptômes de la psychopathologie, est au centre de cette théorie par le biais d'un certain nombre de mécanismes biologiques, psychologiques et sociaux. Si ces relations de cause à effet sont suffisamment fortes, les symptômes peuvent générer un niveau de rétroaction qui les autoentretient. Dans ce cas, le réseau peut rester bloqué dans un état pathologique. La théorie des réseaux considère qu'il s'agit d'une caractéristique générale pour les troubles mentaux et que l'on peut donc comprendre ces derniers comme des états alternatifs stables de réseaux de symptômes fortement connectés. Cette idée conduit naturellement à un modèle général de la psychopathologie, intégrant à la fois un modèle explicatif commun pour les troubles mentaux et une nouvelle définition des concepts associés comme ceux de santé mentale, de résilience, de vulnérabilité et de handicap. En outre, la théorie des réseaux a des implications directes sur la manière de comprendre le diagnostic et le traitement, et elle suggère des pistes prometteuses pour les recherches futures en psychiatrie et dans les disciplines qui lui sont associées.
\end{abstract}

(c) 2020 Publié par Elsevier Masson SAS.
Keywords:

Diagnosis

Mental disorders

Mental health

Network approach

Psychopathology

Resilience

Symptom networks

Treatment

Vulnerability

\begin{abstract}
A B S T R A C T
In recent years, the network approach to psychopathology has been advanced as an alternative way of conceptualising mental disorders. In this approach, mental disorders arise from direct interactions between symptoms. Although the network approach has led to many novel methodologies and substantive applications, it has not yet been fully articulated as a scientific theory of mental disorders. The present paper aims to develop such a theory by postulating a limited set of theoretical principles regarding the structure and dynamics of symptom networks. At the heart of the theory lies the notion that symptoms of psychopathology are causally connected through myriads of biological, psychological and societal mechanisms. If these causal relations are sufficiently strong, symptoms can generate a level of feedback that renders them self-sustaining. In this case, the network can get stuck in a disorder state. The network theory holds that this is a general feature of mental disorders, which can therefore be understood as alternative stable states of strongly connected symptom networks. This idea naturally leads to a comprehensive model of psychopathology, encompassing a common explanatory model for mental disorders, as well as novel definitions of associated concepts such as mental health, resilience, vulnerability and liability. In addition, the network theory has direct implications for how to understand diagnosis and treatment, and suggests a clear agenda for future research in psychiatry and associated disciplines.
\end{abstract}

(c) 2020 Published by Elsevier Masson SAS.

\footnotetext{
Les grands textes de la psychiatrie contemporaine.

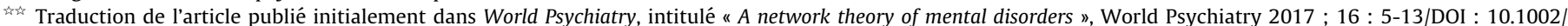

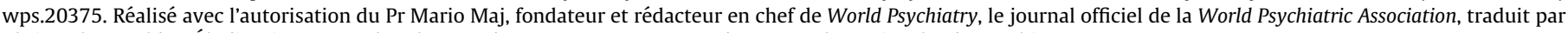
Christophe Gauld et Élodie Giroux avec la relecture de Steeves Demazeux et de Jean-Arthur Micoulaud-Franchi.

Adresse e-mail : jacqueline_parant@orange.fr
} 


\section{Introduction}

Comme toutes les branches de la médecine, la psychiatrie est une discipline avec une approche " centrée sur les problèmes ", fondée et enracinée dans la pratique clinique. Cette pratique s'articule autour d'un ensemble spécifique de problèmes qui amènent les patients à consulter. Par exemple, une personne peut être adressée à un psychiatre parce qu'elle craint que les autres puissent lire dans ses pensées, ce qui provoque chez elle de l'anxiété et un isolement social. Une personne peut également consulter un médecin parce que sa consommation d'alcool nuit à son travail et qu'elle est incapable d'arrêter ou de réduire celle-ci. Une autre personne encore peut avoir développé une anxiété sociale nuisant à sa vie sociale et entraînant des sentiments de solitude et de tristesse. Un objectif important de la psychiatrie (et des disciplines associées telles que la psychologie clinique) consiste à déterminer d'où proviennent ces problèmes et comment les résoudre. L'article présent propose un cadre théorique abordant cette question.

Compte tenu de l'hétérogénéité des problèmes traités par la psychiatrie et la psychologie clinique, il serait peut-être préférable d'utiliser la notion large de " problèmes de vie ». Toutefois, au cours du siècle dernier, la terminologie scientifique a pris une tournure bien différente, et il est donc devenu banal de désigner les personnes aux prises avec ce genre de problèmes comme " souffrant de troubles mentaux ". En conséquence, les problèmes de vie que l'on rencontre dans la pratique clinique ont été classés comme étant des symptômes, comme en témoignent les manuels diagnostiques tels que le DSM-5 et la CIM-10. Par analogie avec la médecine classique, cette utilisation du mot " symptôme " suggère la présence d'une " maladie ", ce qui fournit une réponse orientée à la question de savoir pourquoi certaines personnes souffrent de certains ensembles de symptômes, tandis que ce n'est pas le cas pour d'autres : cela sera expliqué par le fait qu'elles ont un type particulier de maladie, à savoir un trouble mental $[4,29]$.

Cependant, il existe une différence importante entre les troubles mentaux et les maladies. L'utilisation du terme " maladie " implique que l'étiologie soit connue, selon laquelle les symptômes découlent d'un mécanisme pathogène commun, tandis que le terme " trouble mental " fait référence à une constellation syndromique de symptômes qui s'agrègent de manière empirique, souvent pour des raisons inconnues. Malheureusement, pour la quasi-totalité des symptômes des troubles mentaux, les voies pathogènes communes restent difficiles à cerner [4,32,33]. Cette méconnaissance entrave l'application de l'un des schémas explicatifs les plus importants de manière générale en médecine : la recherche des causes communes qui déclenchent les symptômes manifestes [4,29]. Par exemple, si une personne crache du sang, qu'elle a une douleur à la poitrine et qu'elle est essoufflée, un médecin peut supposer la présence d'une tumeur pulmonaire. Une telle tumeur est une anomalie localisée, physiquement identifiable dans le corps, qui agit en tant que cause commune vis-à-vis de la symptomatologie [4]. En conséquence, même si les symptômes sont phénoménologiquement distincts, ils sont homogènes sur le plan causal, car ils sont les effets causaux de la même maladie. Dans ce cas, l'élimination de l'entité pathogène (par exemple, la destruction des cellules cancéreuses par chimiothérapie) supprime la cause commune des symptômes, et ces derniers disparaissent en conséquence. Ce type de stratégie n'a pas été très efficace en psychiatrie, précisément parce qu'aucun mécanisme central de la maladie ni aucune voie pathogène n'ont été identifiés pour les troubles mentaux. La question est désormais de savoir pourquoi une telle identification n'a pas été possible.

Des travaux récents ont émis l'hypothèse que nous ne pouvons pas trouver de mécanisme pathogène central pour les troubles mentaux car un tel mécanisme n'existe pas. En particulier, au lieu d'être des effets d'une cause commune, les symptômes psychiatriques pourraient être causés par les autres [6,17]. Par exemple, si quelqu'un croit qu'autrui peut lire dans ses pensées (idée délirante), cela peut générer une suspicion très importante (paranoïa) ; cette paranoïa peut conduire à éviter tout contact avec d'autres personnes (isolement social), ce qui, du fait que les actions de l'individu ne sont plus corrigées par l'environnement social, peut entretenir et exacerber les idées délirantes déjà présentes. De cette manière, les symptômes peuvent former des boucles de rétroaction qui conduisent la personne à basculer dans un état où ses symptômes sont activés de manière prolongée. C'est cet état qui phénoménologiquement est identifié/reconnu comme un trouble mental $[5,17]$.

Comme les interactions entre les symptômes peuvent être comprises dans la forme d'un réseau, dans lequel les symptômes sont des nœuds et les interactions causales entre les symptômes des connexions entre les nœuds, cette conceptualisation est désormais connue sous le nom d'approche réseau de la psychopathologie. Les recherches méthodologiques menées dans le cadre de cette approche se sont concentrées sur le développement de techniques statistiques dont le rôle est d'identifier, à partir des données empiriques, des structures de réseaux, parmi les symptômes psychiatriques [5,13]. Ces techniques ont aujourd'hui été appliquées à divers construits psychiatriques, tels que la dépression [8,28], les troubles anxieux [2,27], le stress posttraumatique [39], le deuil complexe [46], l'autisme [19,47], les troubles psychotiques [1,31], l'abus de substance [44], la structure générale de la symptomatologie psychiatrique [4], les manuels diagnostiques eux-mêmes $[3,48]$, la qualité de vie liée à la santé [35] et les traits de personnalité [16].

Les résultats de ces études sont encourageants, dans la mesure où, de manière générale, ils concordent avec l'intuition clinique et la théorie établie. Cependant, bien que l'approche réseau ait généré une nouvelle façon de se pencher sur les problèmes de la recherche en psychopathologie, elle n'a pas encore été développée en tant que théorie globale des troubles mentaux. Le but de cet article est de présenter un ensemble de mécanismes explicatifs pouvant être articulés au sein d'un cadre général qui spécifie :

- ce que sont les troubles mentaux ;

- comment ils surviennent ;

- comment ils peuvent être traités de manière optimale.

\section{Réseaux de symptômes}

Le principe central de l'approche réseau de la psychopathologie tient au fait que les troubles mentaux résultent d'une interaction causale des symptômes au sein d'un réseau $[3,18]$. Une telle interaction causale entre les symptômes peut être interprétée à l'aide des théories interventionnistes de la causalité [57]. Dans un tel cadre, la présence d'un lien de causalité signifie que si une intervention (expérimentale ou naturelle) modifie l'état d'un symptôme, cela modifie la distribution de probabilité de l'autre symptôme [43,57]. Il est important de noter que la théorie des réseaux est agnostique quant à savoir comment ces relations causales sont instanciées. Les liens de causalité directe entre les symptômes peuvent reposer sur des processus biologiques (par exemple, insomnie $\rightarrow$ fatigue) ou des processus psychologiques (par exemple, perte d'intérêt $\rightarrow$ sentiment de culpabilité), sur des couplages homéostatiques (par exemple, l'appétit et le sommeil interagissent tous les deux avec l'horloge biologique, en sorte que si l'un est perturbé, il y a de grandes chances que l'autre le soit aussi), sur les normes sociales (par exemple, la dépendance à 


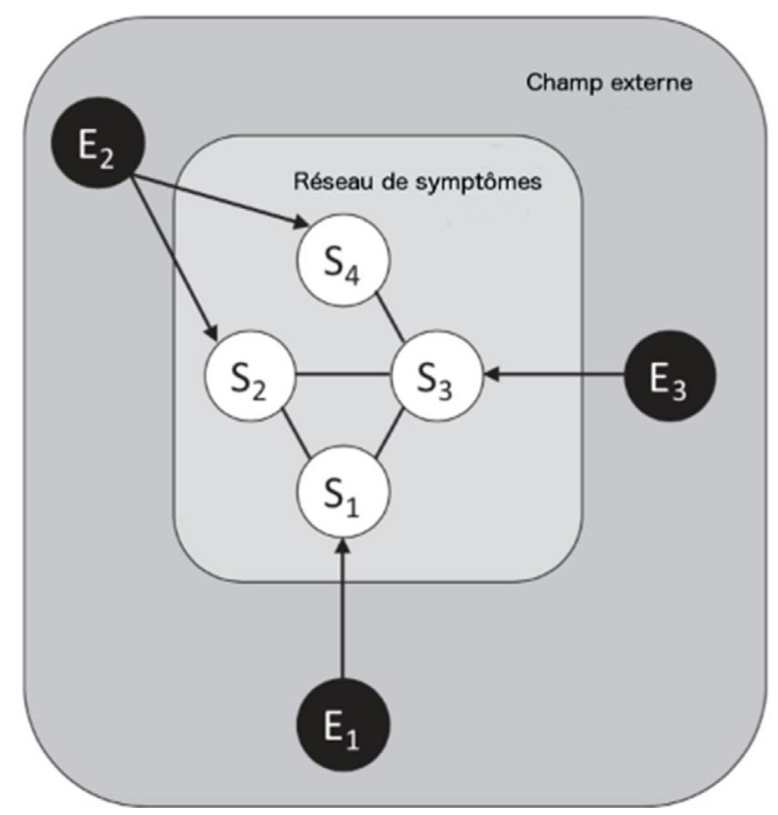

Fig. 1. Un réseau de symptômes composé de quatre symptômes $\left(S_{1}-S_{4}\right)$. Si deux symptômes ont tendance à s'activer l'un l'autre, ils sont reliés par une ligne (par exemple, $\mathrm{S}_{1}-\mathrm{S}_{2}$ ). Les symptômes qui ne sont pas directement connectés les uns aux autres (par exemple, $S_{1}$ à $S_{4}$ ) peuvent toujours se synchroniser s'ils partagent un voisin commun dans le réseau (par exemple, $S_{3}$ ). Les facteurs externes qui affectent le réseau (par exemple, des accidents de la vie) sont représentés dans le champ externe. Ceux-ci peuvent être spécifiques à certains symptômes $\left(E_{1}, E_{3}\right)$ ou ils peuvent être communs à plusieurs symptômes $\left(E_{2}\right)$.

l'héroïne augmente le risque de confrontation avec les forces de l'ordre dans les pays où une telle consommation est interdite par la loi) ou encore sur d'autres processus.

Les schémas d'interaction symptôme-symptôme peuvent être encodés dans une structure en réseau. Dans une telle structure, les symptômes sont représentés par des nœuds. Les nœuds, correspondant à des symptômes qui s'activent directement les uns les autres, sont reliés, tandis que les nœuds correspondant à des symptômes qui ne s'activent pas directement les uns les autres ne le sont pas. Un exemple de structure de réseau est donné à la Fig. 1.

Les facteurs qui peuvent influer les symptômes depuis l'extérieur du réseau (par exemple, les accidents de la vie) constituent le champ externe des symptômes. Tout changement dans le champ externe (par exemple, perdre son conjoint) peut activer les symptômes du réseau (par exemple, une humeur dépressive). À leur tour, ceux-ci peuvent activer des symptômes voisins (comme l'insomnie, la culpabilité, l'anxiété) qui alignent leur état sur le symptôme dépressif. Il faut noter que si les facteurs du champ externe sont en dehors du réseau, ils ne sont pas nécessairement en dehors de la personne [5]. L'inflammation [44], par exemple, est un processus qui se déroule à l'intérieur de la personne, mais dont les effets sur des symptômes comme la fatigue, l'humeur ou l'anxiété proviennent néanmoins de l'extérieur du réseau de symptômes, tant qu'aucun nœud dans le réseau n'est prévu pour l'inflammation. Ainsi, le champ externe est bien externe par rapport au réseau psychopathologique, mais il ne l'est pas par rapport aux limites physiques de la personne. C'est un point important (et très plausible dans certains cas) : il est possible qu'il faille placer dans le champ externe certains fonctionnements cérébraux anormaux qu'on pense communément associés à des troubles mentaux [30] ; par exemple, les délires et les hallucinations pourraient très bien survenir de cette manière.

Si tous les symptômes d'un réseau interagissent les uns avec les autres et que ces interactions ont toutes la même force, alors les symptômes sont dits interchangeables, sauf pour ce qui concerne de leur dépendance au champ externe. Dans une telle configuration, si les connexions sont fortes, les symptômes du réseau afficheront un comportement hautement synchronisé : si un symptôme est actif, il est très probable que les autres symptômes le soient également. Cependant, si tous les symptômes ne possèdent pas d'interaction directe ou si certaines interactions sont beaucoup plus fortes que d'autres, certains symptômes du réseau peuvent être actifs alors que d'autres ne le sont pas. Dans ce cas, la structure du réseau s'organisera en cluster : au sein de l'archipel des symptômes psychopathologiques, nous trouverons des groupes d'îlots particuliers, très proches les uns des autres et qui s'influenceront donc mutuellement à un plus haut degré [3].

Par exemple, l'insomnie est susceptible d'avoir un effet direct et important sur la fatigue, mais un effet beaucoup plus faible sur le sentiment de culpabilité ; si l'insomnie peut avoir une influence sur le sentiment de culpabilité, il est probable que cet effet sera médié par d'autres facteurs, comme, par exemple, une perte d'intérêt ou des problèmes de concentration. De même, la consommation excessive d'alcool affectera d'abord la capacité de l'usager à réaliser ses activités quotidiennes, un symptôme qui déclenchera probablement l'apparition de nouveaux problèmes (par exemple, la perte de son emploi). Si de tels groupes de symptômes forment des sous-réseaux plus étroitement connectés au sein d'un réseau psychopathologique plus vaste, ils se manifesteront sous la forme de configurations fiables de co-activation entre symptômes.

\section{Théories des réseaux}

Les idées présentées ci-dessus peuvent être généralisées sous la forme d'un modèle théorique global de la psychopathologie. En particulier, je défends l'idée que les quatre principes suivants constituent les fondements de la théorie des réseaux pour les troubles mentaux :

- principe 1. Complexité : les troubles mentaux sont mieux caractérisés en termes d'interaction entre composants d'un réseau de symptômes ;

- principe 2. Correspondance composant-symptôme : les composants du réseau en psychopathologie correspondent aux problèmes qui ont été codifiés en tant que symptômes au cours du siècle dernier et qui apparaissent comme tels dans les manuels diagnostiques actuels ;

- principe 3. Connexions causales directes : la structure du réseau est générée par une configuration typique de relations causales directes entre les symptômes ;

- principe 4. Une structure en réseau peut s'appliquer aux troubles mentaux: le réseau de symptômes en psychopathologie présente une topologie non triviale, dans laquelle certains symptômes sont plus étroitement liés que d'autres. Ces regroupements de symptômes donnent lieu à la manifestation phénoménologique des troubles mentaux en tant que groupes de symptômes qui apparaissent souvent ensemble.

Ces principes impliquent que l'étiologie des troubles mentaux peut être envisagée en termes de processus de propagation d'activation au sein d'un réseau de symptômes [3,36,53]. Si un symptôme survient (ce qui peut se produire pour différentes raisons selon la personne, le moment ou le contexte), cela aura une influence sur la probabilité qu'un symptôme connecté survienne également. Ainsi, les ensembles de symptômes couplés et qui sont proches dans la structure du réseau auront tendance à se synchroniser. Les troubles mentaux apparaissent alors, lorsque des groupes de symptômes étroitement liés se maintiennent activement les uns les autres, générant un groupe psychopathologique de symptômes qui s'auto-entretiennent. 
Quelques remarques au sujet de ces principes s'imposent. Le principe 1, la complexité, semble être le moins problématique. À l'exception de quelques rares cas exemplaires [33], aucune cause singulière sur le plan théorique n'a encore pu être identifiée pour un trouble mental ; dès lors, concevoir les troubles mentaux comme des composants qui interagissent au sein d'un système complexe est non seulement plausible, mais c'est d'une certaine manière la seule conception pertinente. Ainsi, ce principe est en accord avec le consensus suivant lequel les troubles mentaux sont multifactoriels dans leur constitution, leur étiologie et le soubassement causal, ce qui semble extrêmement plausible au vu de l'état actuel de la science $[33,41]$.

Le principe 2, la correspondance composant-symptôme, est moins évident. Il part de l'hypothèse que les symptômes en psychopathologie sont définis avec un grain satisfaisant et qu'ils permettent correctement d'identifier les éléments importants du réseau psychopathologique. Dans la mesure où des facteurs non pris en compte dans les systèmes diagnostiques courants peuvent jouer un rôle dans les réseaux de symptômes (par exemple, des processus psychologiques non inclus dans la symptomatologie, ou encore des états neuronaux ou des antécédents génétiques), ils doivent le faire :

- soit en constituant le symptôme en question (par exemple, le symptôme d'anxiété implique certaines activations neuronales dans le cerveau, laquelle constitue en partie ce symptôme) ;

- soit en constituant une connexion symptôme-symptôme (par exemple, l'horloge biologique fait partie du système qui génère la relation insomnie $\rightarrow$ fatigue) ;

- soit en agissant comme une variable appartenant au champ externe (par exemple, une douleur chronique peut jouer un rôle de facteur externe provoquant la fatigue).

Le principe 3, les connexions causales directes, apparaît pertinent pour plusieurs raisons. Premièrement, les systèmes diagnostiques exigent souvent, de manière explicite, la présence d'une connexion symptôme-symptôme pour établir un diagnostic. Deuxièmement, les cliniciens produisent spontanément des réseaux de causalité lorsqu'on leur demande comment les symptômes s'imbriquent $[4,34]$, et les patients en général semblent éprouver peu de difficulté à énumérer les relations de causalité qui existent entre leurs symptômes [22,23]. Troisièmement, les états émotionnels passagers qui sont étroitement liés à la symptomatologie, comme on peut le voir dans les travaux par experience sampling [40], semblent en effet interagir [10,54-56]. Enfin, les analyses en réseaux, portant par exemple sur les symptômes du DSM-5, montrent que de nombreux symptômes restent couplés statistiquement, tout en contrôlant l'effet de tous les autres symptômes [8] ; ceci fournit des preuves, bien qu'indirectes, renforçant l'hypothèse que les symptômes pertinents sont liés de manière causale.

Le principe 4, une structure en réseau peut s'appliquer aux troubles mentaux, soutient que les groupements phénoménologiques stables de symptômes, qui constituent la base des définitions syndromiques actuelles des troubles mentaux, tel que cela est présenté, par exemple, dans le DSM-5, sont de manière générale le résultat de l'architecture causale du réseau de symptômes : les symptômes qui appartiennent à un même trouble sont plus fortement liés de manière causale que les symptômes qui appartiennent à des troubles différents, comme l'illustre la Fig. 2. En conséquence, dans les analyses factorielles de covariance entre symptômes, ou dans les scores totaux définis à partir de ces derniers [12,37], les groupes de symptômes étroitement liés auraient tendance à s'aligner sur le même facteur. Si cela est correct, les analyses factorielles existantes qui étudient la structure de covariance des symptômes peuvent être interprétées comme donnant une première approximation de l'architecture en réseau de symptômes psychopathologiques.

Il découle des principes précédents que la comorbidité est bien une caractéristique intrinsèque des troubles mentaux [17]. En effet, même si les processus d'interaction symptôme-symptôme sont beaucoup plus actifs dans un ensemble donné de symptômes associés à un trouble mental donné, ces processus ne s'arrêtent pas aux frontières d'une catégorie du DSM. Par exemple, si une personne développe une insomnie dans le contexte d'un trouble de stress post-traumatique, cela peut entraîner de la fatigue et des problèmes de concentration - qui sont des symptômes-ponts, lesquels appartiennent également à des réseaux associés à l'épisode dépressif majeur ou au trouble anxieux généralisé - et par conséquent, des motifs de comorbidité au niveau des interactions symptomatiques apparaîtront entre le réseau des épisodes dépressifs majeurs et des troubles anxieux généralisés. Ainsi, au lieu d'être vue comme un frein amené à disparaître avec la sophistication des mesures, une meilleure compréhension de la biologie du cerveau ou encore une meilleure connaissance de la structure génétique des troubles mentaux, la comorbidité peut être considérée comme faisant bel et bien partie de la psychopathologie [17].

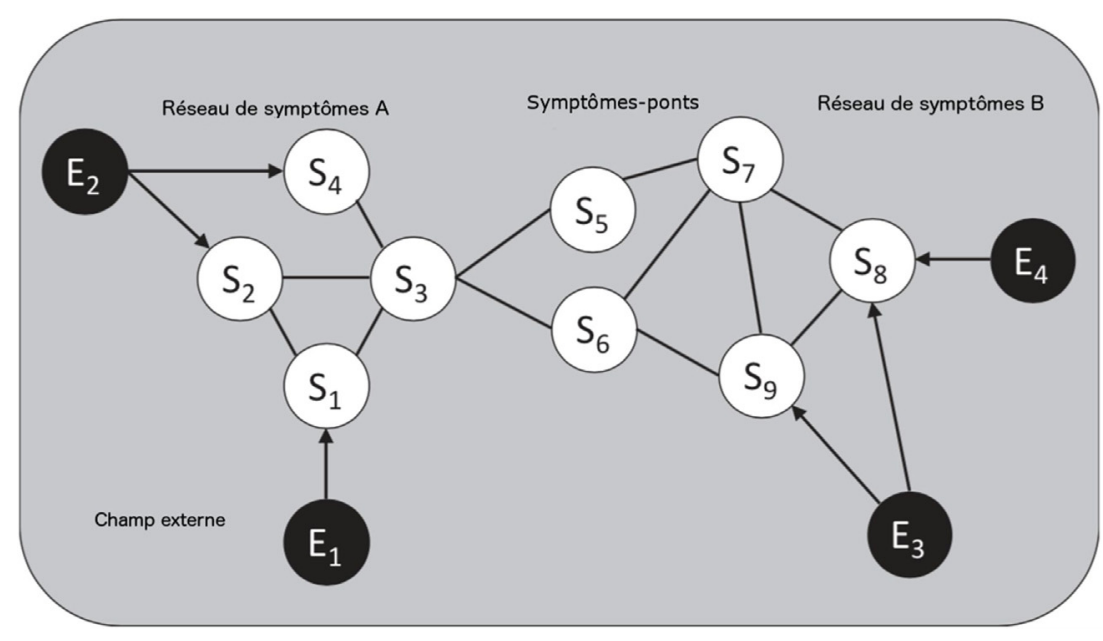

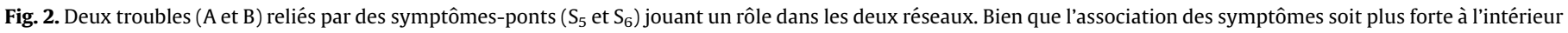
de chaque réseau, un chevauchement entre les troubles est inévitable au sein de la structure du réseau, ce qui fait apparaître un phénomène de comorbidité. 


\section{La dynamique des réseaux de symptômes}

Les implications de l'approche réseau pour ce qui concerne la structure et la comorbidité des troubles mentaux sont évidentes. C'est pourquoi elles ont été rapidement identifiées dès que ce type d'approche a été promu [6,17]. Mais, il a fallu un peu plus de temps pour comprendre que la théorie des réseaux permettait également de mieux rendre compte de la dynamique des troubles mentaux. Le travail de Cramer [18] a joué un rôle déterminant à cet égard, car il a prouvé l'existence d'un phénomène appelé hystérésis dans les paramètres empiriques des réseaux de symptômes [15]. Il s'agit d'une découverte majeure qui pourrait permettre de relier la structure des réseaux de symptômes à leur dynamique.

Pour illustrer l'importance de l'hystérésis, nous devons préciser la manière dont l'étiologie des troubles mentaux se manifeste dans un réseau. La Fig. 3 donne une représentation de ce processus. Supposons que nous partions d'une phase 1 totalement asymptomatique. Dans cette phase, aucun symptôme n'est présent, et les propriétés qui sous-tendent les interactions causales entre les symptômes au cours des phases ultérieures sont en latence, ou dormant (c'est-à-dire, qu'elles sont dispositionnelles, au sens où elles décrivent ce qui pourrait se passer lors de l'activation des symptômes, mais elles ne décrivent pas ce qui se passe au moment présent). Au cours de la phase 2, les événements déclencheurs appartenant au champ externe (par exemple, des accidents de vie) produisent une activation du réseau. En phase 3, l'activation des symptômes se propage à travers le réseau via des connexions entre les symptômes. Dans un réseau de symptômes fortement connecté, les symptômes peuvent entrer en phase 4 , dans laquelle ils se maintiennent mutuellement activés grâce à des phénomènes de rétroaction. En conséquence, le réseau peut se maintenir par luimême et il peut rester actif longtemps après la disparition des événements du champ externe qui avaient déclenché son activation.

Les réseaux fortement connectés présentent ainsi une asymétrie dans leur dynamique : bien que la présence d'un événement déclencheur puisse activer un réseau fortement connecté, l'absence ultérieure de cet événement ne va pas nécessairement le désactiver. C'est le phénomène de l'hystérésis, caractéristique des transitions de phase [51], et qu'on retrouve dans de nombreux systèmes complexes. L'hystérésis est, à mon avis, une caractéristique très plausible des réseaux de symptômes car - dans de nombreux cas en psychopathologie - des événements déclencheurs peuvent causer des problèmes qui perdurent bien après leur disparition. Un exemple important pourrait être l'étiologie du trouble de stress post-traumatique, se développant et persistant après la disparition de l'événement traumatique [39], mais nous observons des exemples similaires dans le développement d'une dépression majeure après la perte d'un conjoint [24] ou encore dans la maltraitance infantile, avec des effets qui persistent longtemps après la fin de la maltraitance [31]. La théorie des réseaux offre donc un mécanisme explicatif de ces phénomènes sous la forme d'une boucle de rétroaction persistante entre symptômes, mécanisme qui est présenté dans un dernier principe :

- principe 5. Hystérésis : les troubles mentaux résultent de la présence d'un phénomène d'hystérésis dans un réseau de symptômes fortement connecté, ce qui implique que les symptômes continuent de s'entre-activer même après la disparition de la cause qui les a déclenchés.

Notons que ces dynamiques ne se produisent que dans des réseaux fortement connectés, car seuls ces réseaux présentent un phénomène d'hystérésis $[15,18]$. Dans les réseaux faiblement connectés, des déclencheurs extérieurs plus importants peuvent provoquer initialement de fortes activations, mais les liens entre les symptômes n'étant pas assez puissants pour les autoentretenir, le réseau se rétablira progressivement et retrouvera son état asymptomatique. Le deuil normal est un processus qui peut être un bon exemple de ce phénomène dans les réseaux de symptômes de la dépression. Un deuil normal peut provoquer un ensemble de symptômes qui ressemble à celui de la dépression caractérisée mais, comme les symptômes ne s'activent pas par rétroaction, cet ensemble de symptômes ne s'auto-entretient pas, de sorte que le système retrouve à terme son état stable et sain. Cette différence est représentée dans la Fig. 4.

Les différentes dynamiques des réseaux de symptômes, en fonction d'une grande variété de paramétrages possibles, suggèrent de nouvelles définitions pour les concepts établis de la recherche en psychopathologie. Premièrement, la notion de santé mentale peut ainsi être définie comme l'état stable d'un réseau faiblement connecté. Notez que cette définition ne revient pas à la définition de la santé mentale comme " absence de symptôme "; bien plutôt, la santé mentale est définie comme un état d'équilibre qu'un système sain est en capacité de retrouver s'il est perturbé. Les réseaux faiblement connectés peuvent toutefois exprimer une symptomatologie en raison de facteurs de stress externes (par exemple, dans le cas du deuil normal) ; à l'inverse, des réseaux fortement connectés peuvent n'exprimer aucune symptomatologie, du moins temporairement, en raison de la suppression locale
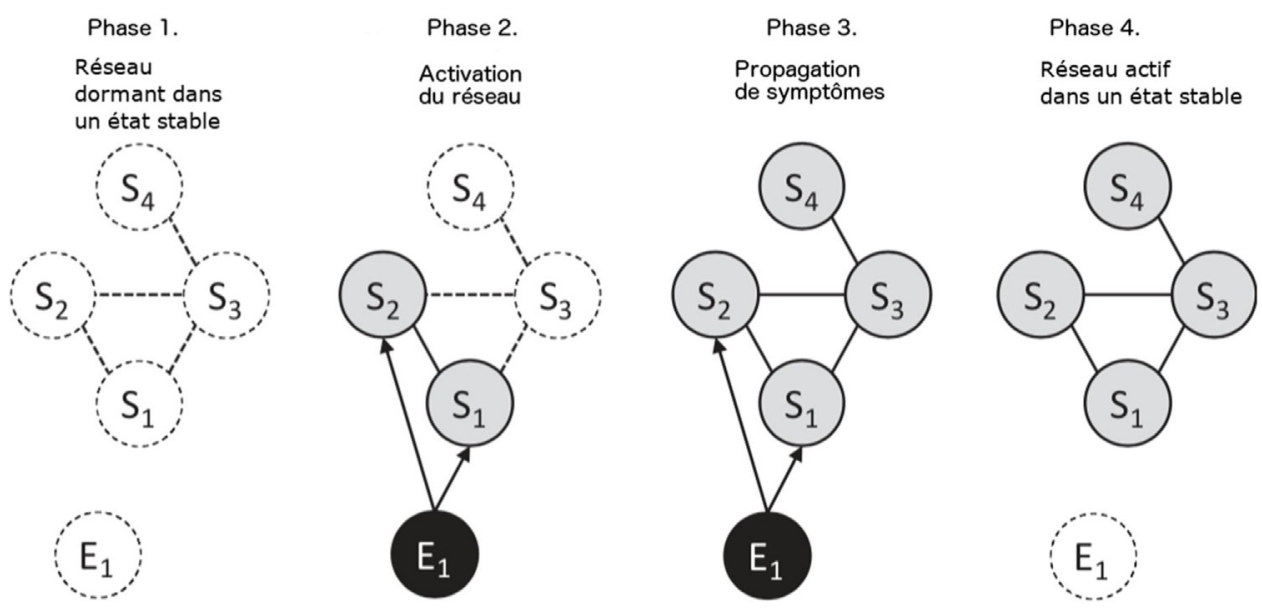

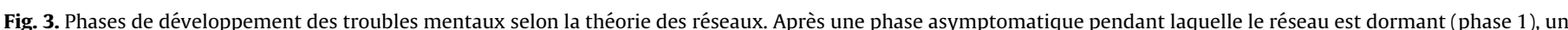

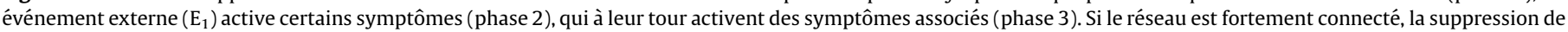
l'événement externe n'entraîne pas de retour à l'état antérieur : le réseau s'auto-entretient et reste bloqué dans son état actif (phase 4). 


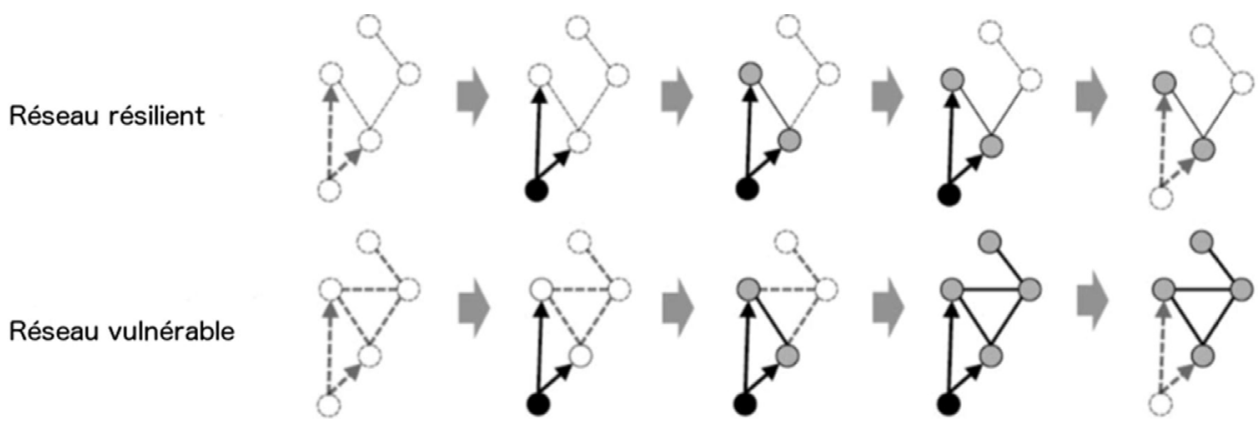

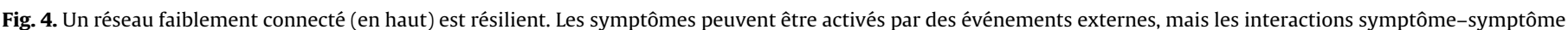

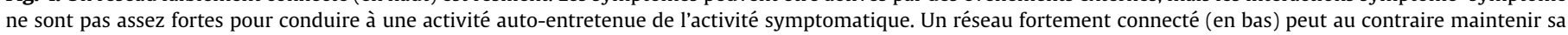
propre activité et évoluer ainsi vers un état pathologique.

de cette symptomatologie (par exemple, une personne présentant un réseau vulnérable impliquant des symptômes psychotiques peut être temporairement asymptomatique en raison de son traitement médicamenteux).

Parallèlement, la notion de trouble mental elle-même est redéfinie en tant qu'état stable (alternatif) d'un réseau fortement connecté, au sens d'un état pathologique qui est distinct, du fait de l'hystérésis, de l'état sain. Le concept de résilience peut être défini comme la disposition des réseaux faiblement connectés à retrouver rapidement un état stable de santé mentale, et le concept de vulnérabilité comme la disposition des réseaux fortement connectés à passer à un état pathologique dès lors qu'une perturbation intervient dans le champ extérieur. Les différences individuelles quant à la possibilité de développer différents types de troubles (par exemple, des troubles d'internalisation versus d'extériorisation) sont expliquées par des différences dans les paramètres du réseau des symptômes correspondants [5]. L'ensemble de ces définitions sont présentées dans le Tableau 1.

\section{Diagnostic et traitement}

Dans la théorie des réseaux, le diagnostic doit être compris comme un processus par lequel un clinicien identifie :

- quels symptômes sont présents ;

- quelles sont les interactions du réseau qui les entretiennent.

On pourrait soutenir que cette démarche est assez proche de la façon dont les cliniciens conçoivent et diagnostiquent habituellement les troubles. Par exemple, les manuels diagnostiques exigent régulièrement de coder non seulement la présence de symptômes, mais également leurs interactions. Ainsi, le diagnostic DSM-5 du trouble obsessionnel-compulsif nécessite non seulement la présence d'obsessions et de compulsions, mais également leur couplage causal (par exemple, une personne est amenée à un nettoyage compulsif en réponse à une obsession de propreté) ; le diagnostic d'un trouble lié à l'usage de substances nécessite l'abandon d'activités importantes à cause de la consommation.

En outre, le DSM-5 contient de nombreuses précisions sur le contexte dans lequel les symptômes devraient apparaître (par exemple, la présence d'insomnie compte uniquement comme symptôme d'épisode dépressif majeur dans le contexte d'une période prolongée d'humeur dépressive et/ou de perte d'intérêt). Enfin, le DSM-5 contient un grand nombre de précisions portant sur des phénomènes de causalité négative, qui exigent l'absence de certaines causes (par exemple, pour ce qui concerne la schizophrénie, l'usage de substances psychoactives en tant que cause des symptômes). Ainsi, bien que le DSM-5 puisse être « théoriquement neutre " par rapport aux autres théories de la psychopathologie [52], il ne l'est pas par rapport à la théorie des réseaux ; au contraire, il donne à travers son appareil de définitions des précisions sur les structures causales des réseaux.

Bien sûr, il existe également des aspects importants de la théorie du réseau que le DSM-5 ignore, comme l'importance de la rétroaction entre les symptômes dans le maintien des troubles mentaux. En outre, les découvertes permises par la théorie des réseaux peuvent générer de nouvelles connaissances sur le rôle fonctionnel et l'importance de symptômes spécifiques dans le maintien de troubles (par exemple, la centralité des symptômes dans le réseau). Ainsi, bien que la théorie des réseaux s'accorde volontiers avec la pratique actuelle en matière de diagnostic, on peut également s'attendre à ce qu'elle l'améliore avec des concepts orignaux et une méthodologie novatrice $[8,13,25]$.

$\mathrm{Si}$ le diagnostic implique l'identification d'un réseau de symptômes, le traitement doit, lui, impliquer de modifier ou de manipuler ce réseau. Du fait de la simplicité des réseaux, ces manipulations peuvent être organisées en seulement trois catégories :

- les interventions sur les symptômes, qui consistent à changer directement l'état d'un ou de plusieurs symptômes ;

Tableau 1

Connectivité du réseau et champ externe.

\begin{tabular}{|c|c|c|c|}
\hline \multirow[t]{4}{*}{ Stresseurs du champ externe } & & \multicolumn{2}{|l|}{ Connectivité du réseau } \\
\hline & & Faible & Forte \\
\hline & $\overline{\text { Faibles }}$ & $\overline{\text { Santé mentale avec haute résilience }}$ & Vulnérabilité élevée (état de rémission possible) \\
\hline & Forts & Symptomatologie importante & Trouble mental \\
\hline
\end{tabular}

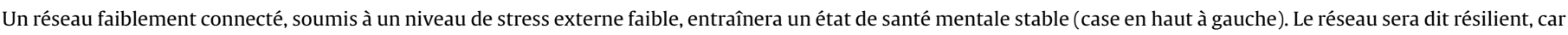

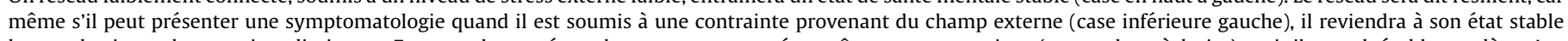

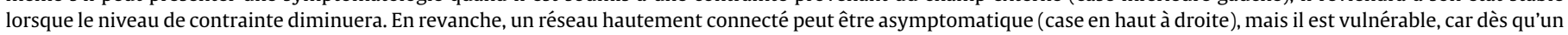
stress est généré dans le champ externe, il peut passer à un état stable de trouble mental (case en bas à droite). 
- les interventions dans le champ externe, qui éliminent un ou plusieurs facteurs déclencheurs ;

- et les interventions sur le réseau, qui modifient la structure même du réseau en modifiant les connexions symptôme-symptôme.

Prenons l'exemple d'un patient psychotique qui présente par ailleurs un trouble de l'usage de substance. Convaincu que d'autres personnes peuvent accéder à ses pensées, il ne sort plus de chez lui et s'isole socialement, ce qui contribue à entretenir ses idées délirantes. Pour ce patient, un exemple d'intervention sur les symptômes peut consister dans la prescription d'un antipsychotique afin de supprimer directement les idées délirantes. Un changement dans le champ externe peut consister à intervenir pour supprimer un ou plusieurs facteurs déclencheurs (par exemple, amener la personne à arrêter sa consommation de substance). Enfin, une intervention sur le réseau peut impliquer une thérapie cognitivo-comportementale, qui vise à ce que la personne apprenne à gérer ses idées délirantes, de sorte que même si celles-ci surviennent parfois, elles ne provoqueront plus un isolement social.

Si les troubles mentaux peuvent effectivement être compris comme des réseaux de symptômes et que le traitement peut être conçu comme on vient de le faire, on pourrait alors coupler une " bibliothèque " d'interventions thérapeutiques à un ensemble de structures en réseaux, cela afin de sélectionner et de planifier de manière optimale ces interventions. Autrement dit, si nous parvenons à détecter la structure en réseau qui régit le type d'interaction symptôme-symptôme pour un individu donné - par exemple, via l'analyse des relations causales perçues [22] ou via les méthodes d'experience sampling [36,40,53] - nous pourrions alors rechercher quelle est la combinaison de stratégies thérapeutiques la plus efficace qui conduit le réseau à rétablir un état sain. Il est probable qu'un traitement efficace nécessite une combinaison d'interventions sur le réseau (afin de rendre l'état de santé accessible) et d'interventions sur les symptômes (pour ramener le système à un état de santé).

\section{Conclusions}

La théorie des réseaux pour les troubles mentaux, telle qu'elle est développée ici, offre un cadre théorique cohérent et clair pour la réflexion en psychopathologie. Les premières étapes empiriques allant dans le sens de cette théorie ont déjà été franchies, sous la forme d'études exploratoires qui dessinent l'architecture en réseau de la symptomatologie $[8,16]$. En supposant que, avec le temps, la structure des réseaux de symptômes devienne de plus en plus précise, la deuxième étape empirique consistera à relier (les différences individuelles dans) l'architecture de ces réseaux à (des différences individuelles dans) des facteurs biologiques, psychologiques et socioculturels pertinents. Enfin, une meilleure compréhension des processus déterminant l'initiation des seuils symptomatologiques et les paramètres de connectivité du réseau devrait nous permettre d'organiser de manière optimale les interventions thérapeutiques existantes et éventuellement d'en développer de nouvelles. Cette possibilité représente un nouveau type d'exploration pour la recherche en psychopathologie, qui, espérons-le, aura plus de succès que les tentatives précédentes pour comprendre et lutter contre les troubles mentaux.

Une question qui se pose est de savoir jusqu'où la théorie estelle capable de généralisation et de quel type de théorie il s'agit. Étant donné que ce modèle en réseaux n'est pas lié à un niveau d'explication en particulier (par exemple, biologique, psychologique ou environnemental) et qu'il ne postule pas de mécanismes particuliers générant la structure du réseau, il est peut-être préférable de l'appréhender comme un cadre d'organisation - un schéma explicatif valant de manière large pour tous les sousdomaines de la psychopathologie. À cet égard, cette théorie rappelle celle de Darwin sur l'évolution, qui fournit également un ensemble de mécanismes explicatifs (par exemple, la mutation, la sélection naturelle, l'adaptation) qui peuvent se déployer de différentes manières selon les espèces. Comme la théorie de l'évolution, la théorie de la psychopathologie fondée sur les réseaux fournit des principes explicatifs généraux (par exemple, l'hyperconnectivité dans les réseaux de symptômes donne lieu à des états stables alternatifs, qui correspondent à des troubles pathologiques), sans spécifier à l'avance les conditions de réalisation ou de mise en œuvre de ces principes. C'est un avantage, car cela signifie que la théorie des réseaux offre un cadre pour l'intégration de différents niveaux d'explication (biologique, psychologique, sociologique), ce qui, à mon avis, est une caractéristique nécessaire de toute théorie fructueuse des troubles mentaux. Dans le même temps, le modèle n'est pas simplement métaphorique : en s'accordant sur quelques hypothèses simplificatrices, la théorie des réseaux peut être représentée sous une forme mathématique $[21,38]$ et elle permet ainsi de simuler à la fois l'évolution des troubles et les effets de diverses interventions thérapeutiques.

Cependant, il reste à explorer dans quelle mesure la théorie des réseaux peut servir de modèle explicatif exhaustif. Il est clair que certains troubles s'y inscrivent mieux que d'autres. Elle fonctionne bien pour des troubles transitoires et pour des troubles chroniques présentant un début relativement bien défini (dépression majeure, trouble de stress post-traumatique, trouble obsessionnel-compulsif, trouble lié à l'usage de substances, trouble panique, trouble anxieux généralisé, phobies, troubles de l'alimentation). Les troubles avec une configuration cyclique (par exemple, un trouble bipolaire) peuvent également être intégrés dans un modèle au sein duquel l'état stable est un cycle plutôt qu'un point fixe. Mais il est moins évident que cette théorie puisse tenir compte de la genèse des troubles qui se développent lentement (par exemple, les troubles du spectre de l'autisme, les troubles de la personnalité, certains aspects de la schizophrénie). Ces troubles sont certes susceptibles aussi de présenter des interactions entre les différents symptômes, mais cela doit en partie impliquer des processus de développement qui se déploient à des échelles de temps très différentes. Par exemple, dans l'autisme, il est probable qu'un symptôme tel que l'évitement du contact visuel limitera, à long terme, la capacité d'un enfant à apprendre les modes d'interaction sociale, ce qui conduira à des difficultés dans le maintien des relations sociales. Cependant, ce processus comprend probablement des processus de rétroaction rapide impliquant le circuit de la récompense de l'interaction sociale, ce qui conduit à un effet " poupée russe » de réseaux dans les réseaux. La question de savoir si de tels troubles mentaux relèvent de la théorie des réseaux et à quoi ressemblerait une telle théorie est donc une question importante pour les recherches futures.

Il est à noter que la théorie proposée dans cet article est très simple. En particulier, le principe 2, la correspondance composantsymptôme, semble assez rigide, mais il existe diverses autres propriétés de la théorie qui, à mesure que la recherche progresse, pourraient bien s'avérer être de fortes idéalisations et abstractions. C'est un choix délibéré. Les réseaux sont par nature assez complexes et, compte tenu de notre ignorance actuelle, je pense qu'il est préférable d'avoir une théorie des réseaux relativement malléable, qu'il faudra peut-être modifier à mesure que les données de la recherche nous parviennent, plutôt que de commencer par un modèle trop compliqué, comportant un ensemble indéfini de variables, qui n'oppose aucune restriction aux données et qui n'entretient pas de relations claires avec les preuves empiriques. Mon espoir est que, grâce aux itérations successives du modèle de réseau de symptômes, nous converge- 
rons finalement vers un modèle acceptable des troubles mentaux qui, même s'il est probablement plus complexe que la formulation actuellement donnée dans cet article, sera toujours suffisamment malléable pour être scientifiquement exploitable.

Enfin, comme le montrent les exemples fournis dans cet article, les liens entre les symptômes sont souvent triviaux. Si vous ne dormez pas, vous vous fatiguez; si vous voyez des choses qui ne sont pas là, vous devenez anxieux ; si vous consommez trop de drogue, vous risquez d'avoir des problèmes avec la police, etc. Il est probable, à mon sens, que ces relations symptôme-symptôme soient ancrées dans des processus biologiques, psychologiques et sociaux très ordinaires (et peuvent donc entraîner des dysfonctionnements préjudiciables dans ces processus [52]). Cette constatation est intéressante, car cela signifie que les troubles ne sont pas des entités éphémères mal comprises, dont la nature devrait être révélée par de futures recherches psychologiques, neuroscientifiques ou génétiques (ce qui semble être une conviction répandue, si ce n'est même la conception dominante parmi les chercheurs). Le fait que nous possédions l'ensemble des symptômes de base et que nous comprenions un grand nombre de leurs relations signifie également que nous disposons déjà d'un modèle d'étude acceptable de ce que sont les troubles et de leur fonctionnement.

Si tel est le cas, notre difficulté actuelle à comprendre les troubles mentaux n'est peut-être pas due à des capacités d'observation limitées, à des instruments de mesure rudimentaires ou à des données inadéquates, comme on le suppose généralement. Au lieu de cela, nous avons peut-être simplement manqué d'un cadre théorique pour organiser les faits empiriques.

\section{Déclaration de liens d'intérêts}

Les auteurs déclarent ne pas avoir de liens d'intérêts.

\section{Références}

[1] Bak M, Drukker M, Hasmi L, et al. An n51 clinical network analysis of symptoms and treatment in psychosis. PLoS One 2016;11:e0162811.

[2] Beard C, Millner AJ, Forgeard MJ, et al. Network analysis of depression and anxiety symptom relationships in a psychiatric sample. Psychol Med 2016;14:1-11.

[3] Borsboom D, Cramer AOJ, Schmittmann VD, et al. The small world of psychopathology. PLoS One 2011;11:e27407.

[4] Borsboom D, Cramer AOJ. Network analysis: an integrative approach to the structure of psychopathology. Annu Rev Clin Psychol 2013;9:91-121.

[5] Borsboom D. Mental disorders, network models, and dynamical systems. In: Kendler KS, Parnas J, editors. Philosophical issues in psychiatry, Vol. 4: Nosology. Oxford: Oxford University Press; 2017.

[6] Borsboom D. Psychometric perspectives on diagnostic systems. J Clin Psychol 2008;64:1089-108.

[8] Boschloo L, van Borkulo CD, Borsboom D, et al. A prospective study on how symptoms in a network predict the onset of depression. Psychother Psychosom 2016;85:183-4.

[10] Bringmann LF, Madeline LP, Vissers N, et al. Assessing temporal emotion dynamics using networks. Assessment 2016;23.

[12] Caspi A, Houts RM, Belsky DW, et al. The p factor: one general psychopathology factor in the structure of psychiatric disorders? Clin Psychol Sci 2014;2:119-37.

[13] Costantini G, Epskamp S, Borsboom D, et al. State of the art personality research: a tutorial on network analysis of personality data in R. J Res Pers 2014;54:13-29.

[15] Cramer AOJ, van Borkulo CD, Giltay EJ, et al. Major depression as a complex dynamic system. PLoS One 2016

[16] Cramer AOJ, van der Sluis S, Noordhof A, et al. Dimensions of normal personality as networks in search of equilibrium: you can't like parties if you don't like people. Eur J Pers 2012;26:414-31.

[17] Cramer AOJ, Waldorp LJ, van der Maas HLJ, et al. Comorbidity: a network perspective. Behav Brain Sci 2010;33:137-93.

[18] Cramer AOJ. The glue of (ab)normal mental life: networks of interacting thoughts, feelings and behaviours. Doctoral dissertation. University of Amsterdam; 2013.

[19] Deserno MK, Borsboom D, Begeer S, et al. Multicausal systems ask for multicausal approaches: a network perspective on subjective well-being in individuals with autism spectrum disorder. Autism 2016.
[21] Epskamp S, Maris G, Waldorp LJ, et al. Network psychometrics. In: Irwing P, Hughes D, Booth T, editors. Handbook of psychometrics. New York: Wiley: 2016.

[22] Frewen PA, Allen SL, Lanius RA, et al. Perceived causal relations: novel methodology for assessing client attributions about causal associations between variables including symptoms and functional impairment. Assessment 2012;19:480-93.

[23] Frewen PA, Schmittmann VD, Bringmann LF, et al. Perceived causal relations between anxiety, post-traumatic stress and depression: extension to moderation, mediation, and network analysis. Eur J Psychotraumatol 2013;4:20656.

[24] Fried EI, Bockting C, Arjadi R, et al. From loss to loneliness: the relationship between bereavement and depressive symptoms. J Abnorm Psychol 2015;124:256-65.

[25] Fried EI, Epskamp S, Nesse RM, et al. What are 'good' depression symptoms? Comparing the centrality of DSM and non-DSM symptoms of depression in a network analysis. J Affect Disord 2016;189:314-20.

[27] Heeren A, McNally RJ. An integrative network approach to Social Anxiety Disorder: the complex dynamic interplay among attentional bias for threat, attentional control, and symptoms. J Anx Disord 2016;42:95-104.

[28] Hoorelbeke K, Marchetti I, De Schryver M, et al. The interplay between cognitive risk and resilience factors in remitted depression: a network analysis. J Affect Disord 2016;195:96-104.

[29] Hyland ME. The origins of health and disease. Cambridge: Cambridge University Press; 2011.

[30] Insel TR, Cuthbert BN. Brain disorders? Precisely. Science 2015;348:499-500.

[31] Isvoranu AM, van Borkulo $C D$, Boyette $L$, et al. A network approach to psychosis: pathways between childhood trauma and psychotic symptoms. Schizophr Bull 2017.

[32] Kendler KS, Zachar P, Craver C. What kinds of things are psychiatric disorders? Psychol Med 2011;41:1143-50.

[33] Kendler KS. The dappled nature of causes of psychiatric illness: replacing the organic-functional/hardware-software dichotomy with empirically based pluralism. Mol Psychiatry 2013;17:377-88.

[34] Kim NS, Ahn W. Clinical psychologists' theory-based representations of mental disorders predict their diagnostic reasoning and memory. J Exp Psychol Gen 2002;131:451-76.

[35] Kossakowski JJ, Epskamp S, Kieffer JM, et al. The application of a network approach to health-related quality of life: introducing a new method for assessing HRQoL in healthy adults and cancer patients. Qual Life Res 2015;25:781-92.

[36] Kramer I, Simons CJP, Wigman JTW, et al. Time-lagged moment-to-moment interplay between negative affect and paranoia: new insights in the affective pathway to psychosis. Schizophr Bull 2014;40:278-86.

[37] Krueger RF. The structure of common mental disorders. Arch Gen Psychiatry 1999;56:921-6

[38] Marsman M, Maris G, Bechger T, et al. Bayesian inference for low-rank Ising networks. Sci Rep 2015;5:9050.

[39] McNally RJ, Robinaugh DJ, Wu GWY, et al. Mental disorders as causal systems: a network approach to post-traumatic stress disorder. Clin Psychol Sci 2014;3:836-49.

[40] Myin-Germeys I, Oorschot M, Collip D, et al. Experience sampling research in psychopathology: opening the black box of daily life. Psychol Med 2009;39:1533-47.

[41] Nolen-Hoeksema S, Watkins ER. A heuristic for developing transdiagnostic models of psychopathology. Explaining multifinality and divergent trajectories. Perspect Psychol Sci 2011;6:589-609.

[43] Pearl J. Causality: models, reasoning and inference. Cambridge: MIT Press: 2000.

[44] Jokela M, Virtanen M, Batty GD. Inflammation and specific symptoms of depression. JAMA Psychiatry 2016;73:1-6.

[46] Robinaugh DJ, Leblanc NJ, Vuletich HA, et al. Network analysis of persistent complex bereavement disorder in conjugally bereaved adults. J Abnorm Psychol 2014;123:510-22.

[47] Ruzzano L, Borsboom D, Geurts HM. Repetitive behaviours in autism and obsessive-compulsive disorder: new perspectives from a network analysis. J Autism Dev Disord 2014;45:192-202.

[48] Tio P, Epskamp S, Noordhof A, et al. Mapping the manuals of madness: comparing the ICD-10 and DSM-IV-TR using a network approach. Int J Methods Psychiatr Res 2016:25:267-76.

[51] van der Maas HLJ, Molenaar PCM. Stagewise cognitive development: an application of catastrophe theory. Psychol Rev 1992;99:395-417.

[52] Wakefield JC. The concept of disorder as a foundation for the DSM's theoryneutral nosology: response to Folette and Houts, Part 2. Behav Res Ther 1999; 10:1001-27.

[53] Wichers M, Geschwind N, van Os J, et al. Scars in depression: is a conceptual shift necessary to solve the puzzle? Psychol Med 2009;40:359-65.

[54] Wichers M, Groot P, Simons CJP, et al. Critical slowing down as a personalised early warning signal for depression. Psychother Psychosom 2016;85:114-6.

[55] Wichers M. The dynamic nature of depression: a new micro-level perspective of mental disorder that meets current challenges. Psychol Med 2014;44:1349-60.

[56] Wigman JTW, Van Os J, Borsboom D, et al. Exploring the underlying structure of mental disorders: cross-diagnostic differences and similarities from a network perspective using both a top-down and a bottom-up approach. Psychol Med 2015;45:1-13.

[57] Woodward J. Making things happen: a theory of causal explanation. Oxford: Oxford University Press; 2003. 


\section{Pour en savoir plus}

[7] Boschloo L, Schoevers RA, van Borkulo CD, et al. The network structure of psychopathology in a community sample of preadolescents. J Abnorm Psychol 2016;125:599-606.

[9] Bringmann LF, Lemmens LHJM, Huibers MJH, et al. Revealing the dynamic network structure of the Beck Depression Inventory-II. Psychol Med 2016;45:74757.

[11] Bringmann LF, Vissers N, Wichers M, et al. A network approach to psychopathology: new insights into clinical longitudinal data. PLoS One 2013;8: e60188.

[14] Cramer AOJ, Borsboom D, Aggen SH, et al. The pathoplasticity of dysphoric episodes: differential impact of stressful life events on the pattern of depressive symptom inter-correlations. Psychol Med 2011;42:957-65.
[20] Epskamp S, Cramer AOJ, Waldorp LJ, et al. qgraph: network visualizations of relationships in psychometric data. J Stat Softw 2012;48:1-18.

[26] Haslbeck J, Waldorp LJ. mgm: Structure Estimation for time-varying mixed graphical models in high-dimensional data. J Stat Softw (2015).

[42] Pe ML, Kircanski K, Thompson RJ, et al. Emotion-network density in major depressive disorder. Clin Psychol Sci 2015;3:292-300.

[45] Rhemtulla M, Fried EI, Aggen SH, et al. Network analysis of substance abuse and dependence symptoms. Drug Alcohol Depend 2016;161:230-7.

[49] van Borkulo C, Boschloo L, Borsboom D, et al. Association of symptom network structure with the course of depression. JAMA Psychiatry 2015;72:121926.

[50] van Borkulo CD, Borsboom D, Epskamp S, et al. A new method for constructing networks from binary data. Sci Rep 2014;4:5918. 